\title{
Magnetic multipole moments (Gauss coefficients) and vector potential given by an arbitrary current distribution
}

\author{
F. J. Lowes ${ }^{1}$ and B. Duka ${ }^{2}$ \\ ${ }^{1}$ School of Chemistry, Newcastle University, Newcastle Upon Tyne, NE1 7RU, U.K \\ ${ }^{2}$ Department of Physics, Faculty of Natural Sciences, Tirana University, Albania
}

(Received November 25, 2009; Revised December 17, 2010; Accepted August 4, 2011; Online published February 21, 2012)

\begin{abstract}
Until recently there has been nothing in the geomagnetic literature giving the Gauss coefficients (equivalent to magnetic multipole moments) for the magnetic scalar potential produced outside a finite-sized region of electric current. Nor has there been an expression for the corresponding magnetic vector potential. This paper presents a simple expression for the Gauss coefficients in terms of a volume integral over the current, and also a series expansion of the vector potential in terms of these coefficients. We show how our result is related to the classical expressions for the scalar potential given by a spherical current sheet, and to the results of the recent papers by Engels and Olsen (1998), Stump and Pollack (1998) and Kazantsev (1999).
\end{abstract}

Key words: Gauss coefficients, magnetic field, magnetic scalar potential, multipole moments, spherical harmonics, vector potential, vector spherical harmonics.

\section{Introduction}

In considering the main geomagnetic field outside the Earth, most workers specify the field by its scalar potential expanded in terms of spherical harmonics, and the corresponding Gauss coefficients, which are scaled versions of the classical multipole moments. There is a similar approach in electrostatics, and many classical texts show how to calculate these moments by integrating over the electric charges (or the equivalent magnetic monopoles) that are the source of the field. However the source of the main geomagnetic field is not monopoles but electric currents, and there did not appear to be in the literature general expressions for calculating the moments (higher than the dipole) by integrating over the current system. Nor were there readily available expressions for the equivalent vector potential distribution. The present paper derives explicit expressions for the Gauss coefficients as integrals over an arbitrary current distribution. It also presents the vector potential analogue of the scalar spherical harmonics, and relates the moments in the two approaches. It compares our results with those of previous workers in a consistent notation.

As is usual, we assume that the source current density $\boldsymbol{J}$ is varying so slowly in time that we can assume that $\operatorname{div} \boldsymbol{J}=0$. We are concerned only with real current density, so ignore the effect of any magnetisation. The rest of this Introduction presents the basic ideas of magnetic scalar and vector potentials, and of toroidal and poloidal fields.

\subsection{Magnetic scalar potential, poles and multipoles}

If the sources of magnetic field are within a limited region $V$, then outside this source region we have curl $\boldsymbol{H}=0$,

Copyright (c) The Society of Geomagnetism and Earth, Planetary and Space Sciences (SGEPSS); The Seismological Society of Japan; The Volcanological Society of Japan; The Geodetic Society of Japan; The Japanese Society for Planetary Sciences; TERRAPUB.

doi:10.5047/eps.2011.08.005 so the resulting magnetic field at the field point $\boldsymbol{r}$ can be expressed as the gradient of a scalar potential,

$$
\boldsymbol{H}(\boldsymbol{r})=-\operatorname{grad} \phi(\boldsymbol{r}) .
$$

In the external region we also have $\operatorname{div} \boldsymbol{B}=\operatorname{div}(\mu \boldsymbol{H})=0$, so in a region of constant permeability $\mu$ the potential $\phi$ is a solution of the Laplace equation $\nabla^{2} \phi=0$.

If the source is a single magnetic pole of strength $p$ (a fictitious, but useful, analogue of an electric charge) at the origin, then we have

$$
\phi=p / 4 \pi r=\text { moment }^{(0)} / 4 \pi r=M^{(0)} / 4 \pi r,
$$

where the choice of notation, degree $l=0$, will be explained later. If we have two poles of strength $+p$ and $-p$, centred at the origin but displaced from each other along the $z$-axis by a distance $\delta z$, the resulting potential is approximately

$$
\phi=(-p \delta z / 4 \pi) \partial(1 / r) / \partial z=(p \delta z / 4 \pi)\left(z / r^{3}\right) .
$$

If we keep the product $p \delta z$ constant at the value $M_{z}^{(1)}$ as we go to the limit $\delta z \rightarrow 0$, we have a (fictitious) degree 1 point-dipole source for which the external potential is

$$
\phi=M_{z}^{(1)}\left(z / 4 \pi r^{3}\right)=M_{z}^{(1)}\left(\cos \theta / 4 \pi r^{2}\right) .
$$

Similarly, taking two $z$-axis dipoles of opposite sign displaced along the $x$-axis would give a $z x$-quadrupole. In general, a degree $l$ multipole has a moment $M^{(l)}$ involving $l$ factors of displacement, and potential falling off with distance as $1 / r^{l+1}$; an arbitrary degree $l$ multipole can be specified in terms of $(2 l+1)$ independent moments.

\subsection{Scalar potential of a distributed source}

If the source is a distribution having magnetic pole density $\rho(s)$ at source point $s$ then at the field point $r$ Eq. (2) 
generalises to

$$
\phi(r)=\frac{1}{4 \pi} \int \frac{\rho(s)}{|\boldsymbol{r}-\boldsymbol{s}|} d V(\boldsymbol{s})
$$

where the integration is over the source region. It is convenient if (5) can be expressed in the form

$$
\phi(\boldsymbol{r})=\frac{1}{4 \pi} \sum_{i} M_{i} R_{i}(\boldsymbol{r})
$$

where the $M_{i}$ are moments that depend only on the source, i.e. on the distribution of $\rho(s)$, and the $R_{i}(\boldsymbol{r})$ are functions that depend only on the position $r$ of the field point; we will see that this corresponds to replacing the actual source distribution $\rho(s)$ by a series of (fictitious) point multipole sources, all at the origin. (Note that if we choose to measure $\boldsymbol{r}$ from a different origin, then in general the moments $M_{i}$ will change; only the first non-zero moment is independent of the choice of origin-see e.g. Raab and De Lange, 2005, section 1.7.) Provided we are content to restrict the field point to being outside a sphere that contains all the sources (so that $r>s_{\max }$ ), we can do this by expanding $1 /|\boldsymbol{r}-\boldsymbol{s}|$ as a power series in $\boldsymbol{s}$. The successive degree $l=0,1,2, \ldots$ terms in this series lead to moments that are integrals over the source in which the source density $\rho(s)$ is (in effect) weighted by $s^{l}$, and the spatial function $R_{i}(\boldsymbol{r})$ decays with distance as $1 / r^{(l+1)}$. However there are several possible choices of power series, and different choices lead to different selections and numbers of moments.

We will use the spherical harmonic addition theorem for $s<r$,

$$
\frac{1}{|\boldsymbol{r}-\boldsymbol{s}|}=\frac{1}{r} \sum_{l=0}^{\infty}\left(\frac{s}{r}\right)^{l} \sum_{m=-l}^{l} S_{l}^{m}\left(\theta_{r}, \lambda_{r}\right) S_{l}^{m}\left(\theta_{s}, \lambda_{s}\right) .
$$

(Throughout our paper we use real $S_{l}^{m}$, and Schmidt seminormalised $P_{l}^{m}(\cos \theta)$, with $S_{l}^{m}(\theta, \lambda)=P_{l}^{m}(\cos \theta) \cos m \lambda$ for positive $m$, and $S_{l}^{m}(\theta, \lambda)=P_{l}^{|m|}(\cos \theta) \sin |m| \lambda$ for negative $m$. These $S_{l}^{m}(\theta, \lambda)$ have mean-square value over the sphere of $1 /(2 l+1)$.) This gives

$$
\begin{aligned}
\phi(\boldsymbol{r}) & =\frac{1}{4 \pi} \sum_{l, m} \frac{1}{r^{l+1}} S_{l}^{m}\left(\theta_{r}, \lambda_{r}\right) \int s^{l} S_{l}^{m}\left(\theta_{s}, \lambda_{s}\right) \rho(\boldsymbol{s}) d V \\
& =\frac{1}{4 \pi} \sum_{l, m} R_{l}^{m}(\boldsymbol{r}) M_{l}^{m}
\end{aligned}
$$

where the spatial functions $R_{l}^{m}(\boldsymbol{r})$ are the harmonic functions (orthogonal over the sphere)

$$
R_{l}^{m}\left(r, \theta_{r}, \lambda_{r}\right)=\frac{1}{r^{l+1}} S_{l}^{m}\left(\theta_{r}, \lambda_{r}\right),
$$

and the moments $M_{l}^{m}$ are given by

$$
M_{l}^{m}=\int s^{l} S_{l}^{m}\left(\theta_{s}, \lambda_{s}\right) \rho(s) d V
$$

The volume integral is to be taken over the whole source region, and (8) is convergent outside the sphere $r=s_{\max }$ that circumscribes the source region. Successive terms of (8) correspond to the potential of a pole $(l=0)$, three dipoles $(l=1)$, five quadrupoles $(l=2)$, etc. at the origin. (In our magnetic case there are no real monopoles, and the series starts with the dipole, $l=1$, terms.)

In the integral of (10), if the volume element $d V$ is put in the form $d s d A$, the volume integral can be organised into a succession of 'surface' integrals over thin spherical shells of thickness $d s$, followed by integration over radius. This gives

$$
\begin{aligned}
M_{l}^{m} & =\int_{s} s^{l} d s \int_{A} \rho(s) S_{l}^{m}(\theta, \lambda) d A \\
& =\int_{s} s^{l+2} d s \int_{\Omega} \rho(s, \theta, \lambda) S_{l}^{m}(\theta, \lambda) d \Omega .
\end{aligned}
$$

(Integrations are always only over the source region, and potentials are expressed only in the external region, so from now on the subscripts $s$ and $r$ will be omitted.) The integration over $\Omega$ is just a surface harmonic analysis giving the $(l, m)$ contribution from that radius. So if at radius $s$ the source density variation is

$$
\rho(s, \theta, \lambda)=\sum_{l, m} \rho_{l}^{m}(s) S_{l}^{m}(\theta, \lambda)
$$

we have

$$
\rho_{l}^{m}(s)=\frac{2 l+1}{4 \pi} \int \rho(s, \theta, \lambda) S_{l}^{m}(\theta, \lambda) d \Omega
$$

and hence

$$
M_{l}^{m}=\frac{4 \pi}{2 l+1} \int \rho_{l}^{m}(s) s^{l+2} d s
$$

Algebraically (10) and (14) are the same whatever the shape of the real source volume; if this is not spherical $\rho(s)$ is simply put to zero as necessary when making the surface harmonic analysis. (There might of course be arithmetic problems in the surface harmonic analysis if $\rho(s)$ is large next to a boundary.)

Using this spherical harmonic approach has the advantage that for a given degree $l$ the series automatically produces the correct number $(2 l+1)$ of independent functions $R_{l}^{m}(\boldsymbol{r})$ (orthogonal over spherical surfaces) and corresponding moments. Alternatively, if $1 /|\boldsymbol{r}-\boldsymbol{s}|$ is expanded using a Cartesian approach, this leads to terms most easily expressed using tensor notation (see e.g. Raab and De Lange, 2005). However, formally this leads to more than $(2 l+1)$ moments/spatial functions, but not all of these are independent (orthogonal); this redundancy is discussed in physical terms in Wikswo and Swinney (1984), and in terms of traceless tensors by Raab and De Lange (2005, section 1.6).

In the context of geomagnetism, it is conventional to assume that $\mu=\mu_{0}$ everywhere, and to include a factor of $\mu_{0}$ in the definition of a different 'potential' $\psi$, giving

$$
\boldsymbol{B}=-\operatorname{grad} \psi \quad \text { and } \quad \nabla^{2} \psi=0 .
$$

It is also conventional to introduce a reference radius $a$, and to scale the moments $M_{l}^{m}$ and spatial functions $R_{l}^{m}$ so that each now has the same dimensions for all degrees $l$. In our notation the solution of (15) used in geomagnetism is

$$
\psi(\boldsymbol{r})=a \sum_{l=1}^{\infty} \sum_{m=-l}^{l} g_{l}^{m}\left(\frac{a}{r}\right)^{l+1} S_{l}^{m}(\theta, \lambda) ;
$$


the $g_{l}^{m}\left(h_{l}^{|m|}\right.$ for negative $\left.m\right)$ are the so-called Gauss coefficients, appropriate to the reference radius $a$. By comparison with (8) we see that

$$
g_{l}^{m}=\left(\mu_{0} / 4 \pi a^{l+2}\right) M_{l}^{m}
$$

\subsection{Magnetic vector potential}

Of course magnetic fields are produced not by poles, but by currents, having current density say $J(s)$. The magnetic field is then given by the Biot-Savart Law

$$
\boldsymbol{B}(\boldsymbol{r})=\frac{\mu_{0}}{4 \pi} \int \frac{\boldsymbol{J}(\boldsymbol{s}) \times(\boldsymbol{r}-\boldsymbol{s})}{|\boldsymbol{r}-\boldsymbol{s}|^{3}} d V
$$

Inside the source region the concept of scalar potential is no longer useful. We can however use the vector potential $\boldsymbol{A}$, with $\boldsymbol{B}=\operatorname{curl} \boldsymbol{A}$, everywhere, and the equivalent equation to $(5)$ is then

$$
\boldsymbol{A}(\boldsymbol{r})=\frac{\mu_{0}}{4 \pi} \int \frac{\boldsymbol{J}(\boldsymbol{s})}{|\boldsymbol{r}-\boldsymbol{s}|} d V
$$

(When putting $\boldsymbol{B}=\operatorname{curl} \boldsymbol{A}$, the gauge of $\boldsymbol{A}$ is not unique; we have selected the Coulomb gauge (see e.g. Jackson, 1975, section 6.4). However this does not affect the use of $\boldsymbol{A}$ here; see the discussion at the end of Section 4.)

It is a standard result (see e.g. Gray (1978, equation (6)), or Backus et al. (1996, pp. 131-132) that (19) leads to (in our notation)

$$
\begin{aligned}
r B_{r}(\boldsymbol{r}) & =-\frac{\mu_{0}}{4 \pi} \int \frac{1}{|\boldsymbol{r}-\boldsymbol{s}|} \operatorname{div}(\boldsymbol{s} \times \boldsymbol{J}(\boldsymbol{s})) d V \\
& =\frac{\mu_{0}}{4 \pi} \int \frac{1}{|\boldsymbol{r}-\boldsymbol{s}|}\left[\boldsymbol{s} \cdot \operatorname{curl}_{h} \boldsymbol{J}(\boldsymbol{s})\right] d V
\end{aligned}
$$

where the subscript $h$ refers to the surface operator.

Outside the source region the same technique of expanding $1 /|\boldsymbol{r}-\boldsymbol{s}|$ as a series can be applied, though of course we now have to use vector algebra. For example, Backus et al. (1996) expanded $1 /|\boldsymbol{r}-\boldsymbol{s}|$ using the spherical harmonic approach, and by comparing the expressions for the resulting radial field terms with those given by the scalar potential (16), derived a general expression for the $g_{l}^{m}$ in terms of integrals involving derivatives of $\boldsymbol{J}(\boldsymbol{s})$

$$
g_{l}^{m}=\frac{\mu_{0}}{4 \pi} \frac{1}{(l+1) a^{l+2}} \int s^{l} S_{l}^{m}(\theta, \lambda) s \cdot \operatorname{curl}_{h} J(s) d V ;
$$

in effect they made a radially weighted spherical harmonic analysis of $\boldsymbol{s} \cdot \operatorname{curl}_{h} \boldsymbol{J}(\boldsymbol{s})$.

However we are looking for a method that avoids taking derivatives of $\boldsymbol{J}$, as this could lead to errors if $\boldsymbol{J}$ was specified numerically. For each $g_{l}^{m}$ it is possible to use vector algebra, and the constraint $\operatorname{div} \boldsymbol{J}=0$, to convert the integrand of (21) into a form using $\boldsymbol{J}$ directly. Unfortunately, working in Cartesian components often leads to integrands involving all three components $J_{x}, J_{y}$ and $J_{z}$; further manipulations using $\operatorname{div} \boldsymbol{J}=0$ have to be used to reduce the integrands to two components. For example for $l=2$, we get

$$
\begin{aligned}
& g_{2}^{0}=\frac{\mu_{0}}{4 \pi a^{4}} \int z\left(x J_{y}-y J_{x}\right) d V \\
& g_{2}^{1}=\frac{\mu_{0}}{4 \pi a^{4}} \frac{\sqrt{3}}{2} \int\left(x^{2}-z^{2}\right) J_{y} d V \\
& g_{2}^{-1}=\frac{\mu_{0}}{4 \pi a^{4}} \frac{\sqrt{3}}{2} \int\left(z^{2}-y^{2}\right) J_{x} d V \\
& g_{2}^{-2}=\frac{\mu_{0}}{4 \pi a^{4}} \frac{\sqrt{3}}{2} \int\left(y^{2}-x^{2}\right) J_{z} d V \\
& g_{2}^{2}=\frac{\mu_{0}}{4 \pi a^{4}} \sqrt{3} \int x y J_{z} d V .
\end{aligned}
$$

Another possible approach is to expand the inverse radius in (19) using a Cartesian Taylor series expansion. Converting to vector notation, we get for the quadrupole $l=2$ term

$$
A^{(2)}=\frac{\mu_{0}}{4 \pi} \int \frac{3(\hat{\boldsymbol{r}} \cdot \boldsymbol{s})^{2}-s^{2}}{2 r^{3}} \boldsymbol{J}(\boldsymbol{s}) d V .
$$

Taking the curl, using considerable vector algebra, applying the constraint $\operatorname{div} \boldsymbol{A}=0$ (equivalent to $\operatorname{div} \boldsymbol{J}=0$ ), and comparing the terms with those given by the scalar potential approach, we can again obtain the expressions (22) to (26) above.

However these are ad hoc process, only feasible for low degree terms. In Section 2 we present a systematic method for obtaining the Gauss coefficients in terms of integrals over $J(s)$ itself, and the corresponding series expansion for the vector potential. In Section 3 we briefly discuss other approaches and recent work, using a consistent notation.

\subsection{Toroidal and poloidal electric currents and mag- netic fields}

When considering the effects of a current distribution in a sphere, it is common practice to separate the 'toroidal' current systems, which have current purely in concentric spherical surfaces and have no radial component, from the 'poloidal' current systems, which do have radial (as well as tangential) components); see e.g. Backus et al. (1996, section 5.3). It is a standard result that toroidal currents produce only poloidal magnetic fields, both inside and outside the region of current, while poloidal current systems produce only toroidal magnetic fields, and these only inside the current region. So we know that the whole of a poloidal current system (the sum of its radial and associated tangential parts) produces no magnetic field outside the source region.

But the middle term of (20) shows that the radial part of such a poloidal current system gives no external field, so it follows that the tangential part must also give no external field. Therefore it does not matter if the tangential part of any poloidal current system is included or not in our calculation; we do not need to make the toroidal/poloidal separation before integration.

Following Engels and Olsen (1998), in the source region we can put

$$
\boldsymbol{B}=\boldsymbol{B}_{\mathrm{tor}}+\boldsymbol{B}_{\text {pol }}=\operatorname{curl} s T+\operatorname{curl} \operatorname{curl} s P,
$$

where $T$ and $P$ are the defining toroidal and poloidal scalar fields. This leads to the corresponding poloidal and toroidal 
currents

$$
\begin{aligned}
\mu_{0} \boldsymbol{J} & =\mu_{0}\left(\boldsymbol{J}_{\mathrm{pol}}+\boldsymbol{J}_{\mathrm{tor}}\right)=\operatorname{curl} \boldsymbol{B}=\operatorname{curl}\left(\boldsymbol{B}_{\mathrm{tor}}+\boldsymbol{B}_{\mathrm{pol}}\right) \\
& =\text { curl curl } \boldsymbol{s} T+\operatorname{curl} \operatorname{curl} \operatorname{curl} \boldsymbol{s} P,
\end{aligned}
$$

(note that it is $\boldsymbol{J}_{\text {tor }}$ that gives $\boldsymbol{B}_{\mathrm{pol}}$ ) which can be put in the form

$$
\mu_{0}\left(\boldsymbol{J}_{\mathrm{pol}}+\boldsymbol{J}_{\text {tor }}\right)=\operatorname{curl} \operatorname{curl} s T+\operatorname{curl} s Q,
$$

where $Q$ is another scalar field.

\section{Series Expansion of the Vector Potential}

In geomagnetism, until recently the vector potential has been used only indirectly, as a means of obtaining expressions for the scalar potential Gauss coefficients. However Kazantsev (1999) produced expressions for the vector potential produced outside an arbitrary current distribution, using vector potential 'moments'. His paper is difficult to follow, so we now give an equivalent approach for the determination of these moments; we also show how they are related to the scalar potential Gauss coefficients.

We saw in Section 1.2 that a particular external scalar potential $S_{l}^{m}(\theta, \lambda) / r^{l+1}$ came from that part of the charge/pole source distribution in a thin spherical shell that was proportional to $S_{l}^{m}(\theta, \lambda)$. Because of the orthogonality of the $S_{l}^{m}(\theta, \lambda)$, the total $(l, m)$ moment could be obtained by (in effect) performing a spherical harmonic analysis of the charge/pole source distribution within each shell, then weighting by $s^{l+2}$ when integrating over radius to give the total moment. What is now described is the equivalent for an arbitrary current distribution. The middle term of (20) shows that (when integrated over the source region) any radial component of current produces no external field, so in our spherical shell we need consider only the tangential components of current. As our basis functions we use the dimensionless surface vector harmonics Kazantsev (1999) called $\boldsymbol{\alpha}_{l}^{m}(\theta, \lambda)$,

$$
\boldsymbol{\alpha}_{l}^{m}(\theta, \lambda)=\operatorname{curl}\left(\boldsymbol{r} S_{l}^{m}(\theta, \lambda)\right)=-\boldsymbol{r} \times \operatorname{grad}_{h} S_{l}^{m}(\theta, \lambda) .
$$

This $-\boldsymbol{r} \times \operatorname{grad}_{h}$ operator is essentially the same as the angular momentum operator $\boldsymbol{L}$ of quantum mechanics. These $\boldsymbol{\alpha}_{l}^{m}(\theta, \lambda)$ have spherical polar components

$$
\boldsymbol{\alpha}_{l}^{m}(\theta, \lambda)=\left(0, \frac{1}{\sin \theta} \frac{\partial S_{l}^{m}(\theta, \lambda)}{\partial \lambda},-\frac{\partial S_{l}^{m}(\theta, \lambda)}{\partial \theta}\right)
$$

and are essentially the 'vector spherical harmonics' used in electromagnetic wave theory (see e.g. Jackson, 1975, section 16.1), and in the separation of toroidal and poloidal current systems; in the notation of Section 1.4 they are toroidal fields. Just as the individual scalar functions $S_{l}^{m}(\theta, \lambda)$ are orthogonal over the sphere (having mean-square value of $1 /(2 l+1))$, so also are the corresponding surface vector functions $\boldsymbol{\alpha}_{l}^{m}(\theta, \lambda)$ (see e.g. Jackson, 1975), which have a mean-square value of $l(l+1) /(2 l+1)$. (Although this orthogonality is not usually stated explicitly in the geomagnetic context, it is a standard result (see e.g. Lowes, 1975) that the tangential vector fields $\operatorname{grad}_{h} S_{l}^{m}(\theta, \lambda)$ are orthogonal for different $(l, m)$; the $\boldsymbol{r} \times$ operator essentially just interchanges the $\theta$ and $\lambda$ components.)
By analogy with the expansion (8) for the scalar potential, we can expand the external vector potential given by a current distribution in the form

$$
\boldsymbol{A}(\boldsymbol{r})=\frac{\mu_{0}}{4 \pi} \sum_{l, m} \frac{1}{r^{l+1}} \boldsymbol{\alpha}_{l}^{m}(\theta, \lambda) m_{l}^{m} .
$$

(Note that, by analogy with the $M_{l}^{m}$ moments we used for the magnetic scalar potential, we use $m_{l}^{m}$ for the corresponding moments for the vector potential; there should not be any confusion with the superscript $m$ used to denote spherical harmonic order.) For a given $(l, m)$ the field $\operatorname{curl}\left[\boldsymbol{\alpha}_{l}^{m}(\theta, \lambda) / r^{l+1}\right]$ given by this vector potential approach, must have the same field geometry as the field $-\operatorname{grad}\left[S_{l}^{m}(\theta, \lambda) / r^{l+1}\right]$ given by a scalar potential approach; the two fields can differ only by a constant factor. It is straightforward to show that

$$
\begin{aligned}
\operatorname{curl}\left(\boldsymbol{\alpha}_{l}^{m}(\theta, \lambda) / r^{l+1}\right) & =\operatorname{curl}\left(-\boldsymbol{r} \times \operatorname{grad}\left(S_{l}^{m}(\theta, \lambda) / r^{l+1}\right)\right) \\
& =-l \operatorname{grad}\left(S_{l}^{m}(\theta, \lambda) / r^{l+1}\right)
\end{aligned}
$$

(a proof is given by Stump and Pollack, 1998, p. 806), so for a given source the vector potential moments $m_{l}^{m}$ are a factor $l$ smaller than the corresponding scalar potential moments $M_{l}^{m}$.

Using the orthogonality of the $\boldsymbol{\alpha}_{l}^{m}(\theta, \lambda)$, we can expand a general volume tangential current distribution $\boldsymbol{J}_{h}(s, \theta, \lambda)$ as the series

$$
\boldsymbol{J}_{h}(s, \theta, \lambda)=\sum_{l, m} J_{l}^{m}(s) \boldsymbol{\alpha}_{l}^{m}(\theta, \lambda)
$$

where

$$
J_{l}^{m}(s)=\frac{1}{4 \pi} \frac{2 l+1}{l(l+1)} \int \boldsymbol{J}(s, \theta, \lambda) \cdot \boldsymbol{\alpha}_{l}^{m}(\theta, \lambda) d \Omega .
$$

(Strictly, these $J_{l}^{m}$ give only the toroidal part of the surface current $\boldsymbol{J}_{h}$; but this is exactly the part that produces external poloidal magnetic field.) It follows from (34) and (46) below that the moments $m_{l}^{m}$ are given by

$$
m_{l}^{m}=\frac{4 \pi}{2 l+1} \int s^{l+2} J_{l}^{m}(s) d s
$$

analogous to (14) for the scalar potential moment. Reversing the argument used to go from (10) to (14),we get

$$
m_{l}^{m}=\frac{1}{l(l+1)} \int \boldsymbol{J}(\boldsymbol{s}) \cdot \boldsymbol{\alpha}_{l}^{m}(\theta, \lambda) s^{l} d V
$$

for an arbitrary source volume, analogous to (10); the extra factor of $1 / l(l+1)$ occurs because the $S_{l}^{m}$ and $\boldsymbol{\alpha}_{l}^{m}$ have different mean-square values. (In (38) $\boldsymbol{J}(\boldsymbol{s})$ can be the full (toroidal + poloidal) current density; the scalar product with $\boldsymbol{\alpha}_{l}^{m}(\theta, \lambda)$ picks out only the toroidal part.) So from (17) and (37) we get

$$
g_{l}^{m}=\frac{\mu_{0} l}{(2 l+1)} \int\left(\frac{s}{a}\right)^{l+2} J_{l}^{m}(s) d s .
$$

From (17) and (38),

$$
g_{l}^{m}=\frac{\mu_{0}}{4 \pi a^{2}} \frac{1}{(l+1)} \int\left(\frac{s}{a}\right)^{l} \boldsymbol{J}(\boldsymbol{s}) \cdot \boldsymbol{\alpha}_{l}^{m}(\theta, \lambda) d V .
$$


We can then write (33) in the form

$$
\boldsymbol{A}(\boldsymbol{r})=a \sum_{l=1}^{\infty} \frac{1}{l}\left(\frac{a}{r}\right)^{l+1} \sum_{m=-l}^{m=l} g_{l}^{m} \boldsymbol{\alpha}_{l}^{m}(\theta, \lambda),
$$

giving the vector potential directly in terms of the Gauss coefficients; this is the equivalent of the scalar potential expression (16).

A result equivalent to (40) was obtained by Gray (1978) by applying unspecified vector algebra to (21). He also in effect gave (41).

The anonymous referee has shown that (21) can be put in the alternative forms

$$
\begin{aligned}
g_{l}^{m}= & \frac{\mu_{0}}{4 \pi} \frac{1}{(l+1) a^{l+2}} \\
& \times \int \operatorname{grad}_{h}\left(s^{l} S_{l}^{m}(\theta, \lambda)\right) \cdot(\boldsymbol{s} \times \boldsymbol{J}(\boldsymbol{s})) d V
\end{aligned}
$$

and

$$
\begin{aligned}
g_{l}^{m}= & \frac{\mu_{0}}{4 \pi} \frac{1}{(l+1) a^{l+2}} \\
& \times \int \operatorname{curl}_{h}\left(s^{l} S_{l}^{m}(\theta, \lambda) s\right) \cdot \boldsymbol{J}(s) d V .
\end{aligned}
$$

Equation (43) is equivalent to

$$
g_{l}^{m}=\frac{\mu_{0}}{4 \pi} \frac{1}{(l+1) a^{l+2}} \int s^{l} \operatorname{curl}_{h}\left(S_{l}^{m}(\theta, \lambda) s\right) \cdot \boldsymbol{J}(s) d V
$$

which is the same as our (40).

\section{Other Approaches}

Using the notation of this paper, we now summarise the relevant parts of an old classical approach, and of three recent papers.

\subsection{Magnetic field produced outside a spherical sur- face current distribution-Chapman and Bartels (1940)}

Workers on ionospheric phenomena needed to calculate the magnetic field produced by the ionospheric current system, which is often approximated by a surface current distribution. The standard approach (see e.g. Chapman and Bartels, 1940, p. 630, but note that their factor of $4 \pi / 10$ is not needed when working in SI units) is not to work in terms of the current itself, but to specify the current flowing in a spherical surface by the equivalent scalar 'current function' $F(\theta, \lambda)$ (analogous to the 'stream function' of hydrodynamics), that can be expressed as contours on the sphere. This current function is such that between the two contours $F$ and $F+\Delta F$ there is a current flow of $\Delta F$, parallel to the contours, clockwise round a minimum. If a surface harmonic analysis is performed on the $F(\theta, \lambda)$ at radius $s$ to give

$$
F(\theta, \lambda)=\sum_{l, m} I_{l}^{m} S_{l}^{m}(\theta, \lambda)
$$

then the external field produced by the $I_{l}^{m}$ component has the scalar potential

$$
\phi_{l}^{m}(r, \theta, \lambda)=\frac{l}{2 l+1} I_{l}^{m}\left(\frac{s}{r}\right)^{l+1} S_{l}^{m}(\theta, \lambda),
$$

corresponding to a Gauss coefficient

$$
g_{l}^{m}=\mu_{0} \frac{l}{2 l+1}\left(\frac{s}{a}\right)^{l+2} I_{l}^{m} .
$$

In fact for a given current function $F(\theta, \lambda)$ the actual surface current density is

$$
i(\theta, \lambda)=\operatorname{curl}[\hat{\boldsymbol{r}} F(\theta, \lambda)]=-\hat{\boldsymbol{r}} \times \operatorname{grad}_{h} F(\theta, \lambda) .
$$

Comparing this with the definition of $\boldsymbol{\alpha}_{l}^{m}(\theta, \lambda)$ in (31) we see that the surface current distribution corresponding to the current function $I_{l}^{m} S_{l}^{m}(\theta, \lambda)$ at radius $s$ is just

$$
\boldsymbol{i}_{l}^{m}(\theta, \lambda)=i_{l}^{m} \boldsymbol{\alpha}_{l}^{m}(\theta, \lambda) \quad \text { with } \quad i_{l}^{m}=\left(I_{l}^{m} / s\right) .
$$

Expressing (47) in terms of the surface current coefficient $i_{l}^{m}$ gives

$$
g_{l}^{m}=\mu_{0} \frac{l}{2 l+1}\left(\frac{s}{a}\right)^{l+2} s i_{l}^{m} .
$$

The concept of a current function used here is formally only applicable to a surface current (having no component normal to the surface), or to a current sheet thin enough that it is sufficiently accurate to use the thickness-integrated current density as an equivalent surface current; it cannot be applied to a general 3-dimensional current system. However no external magnetic field is produced by any radial component of current (Eq. (20)), so there is no reason why equations such as (46) or (47) should not be integrated over radius, to give the integrated effect of the tangential component of a 3-dimensional current as in (39). But workers using this Chapman and Bartels' approach do not appear to have seen this possibility.

\subsection{Toroidal currents and poloidal magnetic fields-} Engels and Olsen (1998)

The approach of these authors starts with Eq. (30):

$$
\mu_{0}\left(J_{\text {pol }}+J_{\text {tor }}\right)=\operatorname{curl} \operatorname{curl} s T+\operatorname{curl} s Q .
$$

Comparing the $\operatorname{curl} s Q$ toroidal part of this with (48) we see that $Q(s, \theta, \lambda)$, the toroidal current scalar at radius $s$, is simply a scaled version of $F(s, \theta, \lambda)$, the current function at radius $s$. The current function approach used to calculate ionospheric and similar magnetic fields is essentially the same as the toroidal current/poloidal field relationship used in geodynamo theory and elsewhere, and Engels and Olsen explicitly note the identity for the case of a current sheet. In each case, the current function $F$ (or the toroidal scalar $Q$ ) is subject to surface harmonic analysis at a given radius, and the resulting partial coefficient is then weighted by $s^{l+2}$ and integrated over radius (though this last step does not appear to have been done previously by workers using the current function approach). Using the notation of the present paper, for a given radius $s$, Engels and Olsen analysed $\boldsymbol{J}_{\text {tor }}$ in the form

$$
\mu_{0} \boldsymbol{J}_{\mathrm{tor}}(s, \theta, \lambda)=\frac{1}{a} \sum_{l, m} q_{l}^{m}(s) \alpha_{l}^{m}(\theta, \lambda)
$$

They worked in terms of the scalar potential, and used a Green's function approach to show that if the coefficients 
$q_{l}^{m}(s)$ are known as a function of radius $s$, the resulting external field corresponds to a Gauss coefficient

$$
g_{l}^{m}=\frac{l}{2 l+1} \frac{1}{a} \int_{s}\left(\frac{s}{a}\right)^{l+2} q_{l}^{m}(s) d s
$$

equivalent to our (39). In their examples they used standard numerical methods to estimate the coefficients at a succession of discrete radii, and then to estimate the $g_{l}^{m}$. By going to sufficiently high order (and using also the internal fields that we have not considered) they had no problem in applying the method to give the field from thin field-aligned currents joining the north and south auroral zones.

\subsection{Series expansion for vector potential-Kazantsev (1999)}

Kazantsev introduced the approach of analysing the tangential current density in terms of the $\boldsymbol{\alpha}_{l}^{m}(\theta, \lambda)$ and then integrating over radius to give our moments $m_{l}^{m}$. (Note that his moments $j_{l}^{m}$ are numerically $l$ times larger than our $m_{l}^{m}$ ). Unfortunately his notation and mathematical approach are difficult to follow for readers with a geophysical background. Kazantsev did not relate his moments to the Gauss coefficients, and although he gives explicit expressions for the $\boldsymbol{\alpha}_{l}^{m}$, he does so using Cartesian components, which adds further complication.

\subsection{The paper by Stump and Pollack (1998)}

Unlike Engels and Olsen (1998) and Kazantsev (1999), the paper by Stump and Pollack (1998), was restricted to the calculation of the field produced outside the current distribution on a single surface, and they apparently did not know of the Chapman and Bartels (1940) current function approach. As in the other two papers they expanded the current density at radius $s$ in the form (their vector spherical harmonics had the opposite sign)

$$
\boldsymbol{i}(\theta, \lambda)=-\sum_{l, m} k_{l}^{m} \boldsymbol{\alpha}_{l}^{m}(\theta, \lambda)
$$

used the spherical harmonic expansion of $1 /|\boldsymbol{r}-\boldsymbol{s}|$ to give a series representation of the vector potential in the form

$$
\boldsymbol{A}(\boldsymbol{r})=\mu_{0} a \sum_{l, m} k_{l}^{m} \frac{1}{2 l+1}\left(\frac{s}{r}\right)^{l+1} \boldsymbol{\alpha}_{l}^{m}(\theta, \lambda),
$$

and used the result (34) to give

$$
g_{l}^{m}=-\mu_{0} \frac{l}{2 l+1}\left(\frac{s}{a}\right)^{l+2} k_{l}^{m},
$$

equivalent to (47) above.

\section{Conclusion}

In geomagnetism, once the Gauss coefficients are known, it has been usual to calculate the external field using the corresponding scalar potential

$$
\psi(\boldsymbol{r})=a \sum_{l, m} g_{l}^{m}\left(\frac{a}{r}\right)^{l+1} S_{l}^{m}(\theta, \lambda),
$$

but there does not seem to have been a simple expression enabling the Gauss coefficients to be derived by the volume integration over an arbitrary current distribution. We show that

$$
g_{l}^{m}=\frac{\mu_{0}}{4 \pi a^{2}} \frac{1}{(l+1)} \int\left(\frac{s}{a}\right)^{l} \boldsymbol{J}(s) \cdot \boldsymbol{\alpha}_{l}^{m}(\theta, \lambda) d V,
$$

where the vector harmonics $\boldsymbol{\alpha}_{l}^{m}(\theta, \lambda)$ are defined in (31) or (32). If the horizontal part of the current density at radius $s$ is expanded in the form

$$
\boldsymbol{J}_{h}(s, \theta, \lambda)=\sum_{l, m} J_{l}^{m}(s) \alpha_{l}^{m}(\theta, \lambda),
$$

we have

$$
g_{l}^{m}=\frac{\mu_{0} l}{(2 l+1)} \int\left(\frac{s}{a}\right)^{l+2} J_{l}^{m}(s) d s .
$$

There might be situations where it is more convenient to work in terms of the vector potential, and the present paper leads to the simple expression

$$
\boldsymbol{A}(\boldsymbol{r})=a \sum_{l, m} g_{l}^{m} \frac{1}{l}\left(\frac{a}{r}\right)^{l+1} \boldsymbol{\alpha}_{l}^{m}(\theta, \lambda),
$$

giving the external vector potential in terms of the Gauss coefficients and the surface vector harmonics $\boldsymbol{\alpha}_{l}^{m}(\theta, \lambda)$. (Of course when using vector potential, possible gauge transformations mean that in a particular situation different approaches might give different $\boldsymbol{A}(\boldsymbol{r})$; however the difference will simply be the gradient of a scalar function, and will not affect the resultant field.)

Acknowledgments. We thank Angelo De Santis and an anonymous referee for helpful suggestions.

\section{References}

Backus, G., R. Parker, and C. Constable, Foundations of Geomagnetism, Cambridge University Press, 1996.

Chapman, S. and J. Bartels, Geomagnetism, Oxford University Press, 1940.

Engels, U. and N. Olsen, Computation of magnetic fields within source regions of ionospheric and magnetospheric currents, J. Atmos. Sol.-Terr. Phys., 60, 1585-1592, 1998.

Gray, C. G., Multipole expansion of electromagnetic fields using Debye potentials, Am. J. Phys., 46(2), 169-179, 1978.

Jackson, J. D., Classical Electrodynamics, 2nd Ed., Wiley, New York, 1975.

Kazantsev, V. P., Spherical magnetic multipole moments systems of currents, Russ. Phys. J., 42(10), 916-921, 1999. (Translated from Izvestiya Vysshikh Uchebnykh Zavedenii, Fizika, No. 10, pp. 66-72, Oct. 1999.)

Lowes, F. J., Vector errors in spherical harmonic analysis of scalar data, Geophys. J. R. Astron. Soc., 42(2), 637-651, 1975.

Raab, R. E. and O. L. De Lange, Multipole Theory in Electromagnetism, Clarendon Press, Oxford, 2005.

Stump, D. R. and G. L. Pollack, A current sheet model for the Earth's magnetic field, Am. J. Phys., 66(9), 802-810, 1998.

Wickswo, J. P. and K. R. Swinney, A comparison of scalar multipole expansions, J. Appl. Phys., 56(11), 3039-3049, 1984.

F. J. Lowes (e-mail: f.j.lowes@ncl.ac.uk) and B. Duka 